\title{
Nivel de adaptación al contexto táctico en futbolistas juveniles Level of adaptation to the tactical context in youth football players
}

\author{
*Santiago Gaviria Echavarría, *Mateo Sepulveda Arango, *Santiago Sepúlveda Arango, *,**W ilder Geovanny \\ Valencia Sánchez, * Jose Albeiro Echeverri Ramos \\ *Universidad de Antioquia (Colombia), **Asociación Colombiana de Futbolistas Profesionales (Colombia)
}

\begin{abstract}
Resumen. El objetivo del estudio fue describir el nivel de adaptación al contexto táctico en los principios operacionales de conservar, progresar el balón y finalizar en jugadores de fútbol juvenil entre el primer y segundo tiempo de juego. El diseño fue no experimental-descriptivo con participación de 16 futbolistas ( $E d a d M e=14, R I=1$ años; estaturax=165, DE $=.06 \mathrm{~cm}$; $\operatorname{masax}=55.29, D E=5.21 \mathrm{Kg}$; experienciadeportivax $=7.84, \mathrm{DE}=1.34$ años). Seutilizó paralarecolección delainformación la Herramienta de Evaluación del Rendimiento de Juego valido y confiable para esta edad ( $\alpha=.97$ ) a través de un juego modificado de fútbol con una estructura $5+$ portero vs $5+$ portero. Los datos se resumen con proporciones e intervalos de confianza. En el análisis bivariado se estableció diferencias de proporciones entreel primer y segundo tiempo medianteel test de McNemar para datos emparejados. Se registraron en total 191 unidades de toma de decisión con un promedio de porcentaje de efectividad en los principios del $79.48 \%$. Los resultados mostraron que el principio de conservar tuvo un descenso en el segundo tiempo con relación al primero $(-2.26 \%)$, sin implicar diferencias significativas $(p=.16)$. Por otra parte, en el principio de progresar el balón tuvo mayor rendimiento en el segundo tiempo (37.33\%) encontrando diferencias significativas entre ambos tiempos ( $p<.01$ ); el principio de finalizar registró una diferencia del $33.33 \%$ a favor del segundo tiempo. En la efectividad total de la adaptación al contexto táctico se encontró un mayor rendimiento en el segundo tiempo $(24.37 \%)$ con diferencias estadísticamente significativas ( $p<.01)$. El estudio concluye que los futbolistas tienen una mejor adaptación al contexto táctico en el segundo tiempo que en el primer tiempo en el juego.
\end{abstract}

Palabras clave: Fútbol, táctica, principios operacionales, toma de decisión.

\begin{abstract}
The objective of the study wasto describethe level of adaptation to the tactical context in the operational principles of maintaining possession of the ball, penetrating the defense, and attacking the goal in youth soccer players between the first and second half of the game. The design was non-experimental-descriptive with the participation of 16 soccer players (Age $M e=14, I R=1$ years; height $x=165, S D=.06 \mathrm{~cm}$; mass $x=55.29, S D=5.21 \mathrm{~kg}$; sport experience $x=7.84, S D=1.34$ years). The valid and reliable $G$ ame Performance Eval uationTool for this age ( $\alpha=.97$ ) was used to collect the information through a modified soccer game with a structure $5+$ goalkeeper vs $5+$ goalkeeper. Data are summarized with proportions and confidence inter vals. The bivariate analysis, differences of proportions was established between the first and second time using the $\mathrm{McN}$ emar test for paired data. A total of 191 decision-making units were registered with an average percentage of effectiveness in the principles of $79.48 \%$. The results showed that the principle of conserving had a decrease in the second half in relation to the first $(-2.26 \%)$ without implying significant differences $(p=.16)$. On the other hand, the principle of progressing it had a greater performance in the second time (37.33\%) finding significant differences between both times ( $p<$ .01); in the principle of finishing there is a 33.33\% difference between the times. In the total effectiveness of the adaptation to the tactical context, agreater performance was found in the second time (24.37\%), finding statistical ly significant differences $(p<.01)$. The study concludes that soccer players have a better adaptation to the tactical context in the second half than in the first half of the game.
\end{abstract}

Keywords: Football, tactics, operational principles, decision-making.

\section{Introducción}

En los deportes de cooperación-oposición los juga dores deben disponer de habilidades para interactuar eficazmente con oponentes y/ o compañeros (Riera, 2007). Específicamente en el fútbol, la maestría técni-

Fecha recepción: 25-09-20. Fecha de aceptación: 12-12-20 W ilder Geovanny Valencia Sánchez wilder.valencia@ udea.edu.co co-táctica de los jugadores, la dinámica y funcional idad colectiva juegan un papel preponderante en el rendimiento deportivo (Abad, 2010), siendo los principios tácticos un marco de referencia que guía el comporta miento de los jugadores dentro del terreno, haciendo énfasis en la conducta ideal del deportista en ciertas situaciones de juego y que parten de presupuestos teóricos y la lógica del juego (Costa et al., 2009; Garganta \& Pinto, 1997).

El fútbol exige al deportista seleccionar la acción más apropiada para solucionar las situaciones complejas 
que se presenten en la confrontación directa con el rival tanto en duelos individuales o grupales con o sin balón, por medio de la ejecución de las habilidades técnicas y en consideración del contexto táctico (Aguero et al., 2015; García et al. , 2011; Garganta \& Pinto, 1997). Es así, que los principios tácticos han sido utilizados de manera regular en el entrenamiento (Gómez \& Álvaro, 2002), desde edades tempranas por medio de juegos y en situaciones adaptadas en diferentes contextos (González et al., 2011) influyendo en las habilidades técnico-tácticas y capacidades físicas de los jugadores (Arrieta et al., 2017; Sgrò et al. , 2018).

Los principiostácticos son un conjunto de normas de juego, las cuales permiten al jugador solucionar rápida mente problemas que se presentan en el juego (Garganta \& Pinto, 1997). Estos principios de juego son representaciones primordiales en el fútbol, que tienen dos objetivos fundamentales; disponer al jugador para solucionar los problemas tácticos que se presentan en el transcurso de un partido y establecer en el jugador una idea de juego, lo cual suministra un mayor repertorio de posibles soluciones táctico-técnicas y posiblemente afectelainteligenciadejuego (M orales\& M urillo, 2013). Los principios tácticos, ayudan al desarrollo del jugador en la medida que logran una correcta toma de decisiones y una adecuada aplicación práctica durante la competencia, en coherencia con los planteamientos del entrenador (Amado \& Vivas, 2014; Castro et al., 2019; Guimaraes, 2000) entendiendo el sistema, la filosofía de juego y participando en las fases del juego de ataque y defensa con la misma intensidad independiente de la posición (D elgado et al., 2019).

Por lo cual, el nivel táctico relacionado con los principios ha sido un tema de interés de diversos estudios, especialmente en jugadores de fútbol desde los siete años hasta los quince años; donde se analiza el rendimiento en cada categoría, (Blomqvist et al., 2005; Da Silva et al., 2015; González-Víllora et al., 2012; O livares, et al., 2011) el desempeño en diferentes organizaciones de juegos (O livares et al., 2011), se compara expertos y novatos (del Campo et al., 2011), y se analiza los efectos de entrenamiento en la adaptación al contexto táctico (Práxedes et al., 2019; Vergara, 2012).

De esta manera, es importante conocer el nivel de rendimiento táctico para mejorar los procesos deportivos de acuerdo al nivel de toma de decisión de los juga dores mediante estímulos específicos. Esto representa una valiosa información para el entrenador en la perspectiva de la planificación en busca de mejorar el rendimiento deportivo; siendo un tema de interés en los úl-
timosaños(Américo et al. , 2017; Blomqvist et al. , 2005; Correia da Silva et al., 2019; Da Silva et al., 2015; O livareset al., 2011; Vergara, 2012). Esto paraoptimizar el desarrollo de contenido táctico-técnicos en el proceso de entrenamiento (Bettega et al., 2018). Además, los datos de cada estudio aportan valores de referencia en cada categoría, siendo indicadores de rendimiento táctico para la progresión de enseñanza en los contenidos de aprendizaje táctico; a la vez, permite identificar fases sensibles de entrenamiento (López, 2008) relacionados con cada principio de juego y permite la identificación de talentos en el fútbol (O livares et al., 2020).

En consecuencia, este estudio se centra en el análisis de los principios denominados operacionales ofensivos (conservación individual y colectiva de la pelota, progresión del equipo y la finalización de la jugada) (Bayer, 1986), mediante un instrumento que permite realizar una evaluación de la adaptación al contexto táctico, es decir, permite identificar el problema táctico que el jugador enfrenta y determinar si la acción tiene la intención de resolverlo (López \& del Campo, 2018) pudiendo establecer desempeños en el juego por periodos de tiempo.

Por lo anterior, el objetivo del estudio fue describir el nivel de adaptación al contexto táctico en los principios operacionales de conservar, progresar el balón y finalizar en jugadores de fútbol juvenil, y establecer diferencias de rendimiento entre el primer y segundo tiempo del juego.

\section{Material y método}

\section{D iseño}

Estudio no experimental descriptivo donde no se manipularon variables y solo hubo un momento de medición (Hernández et al., 2014).

\section{Participantes}

La muestra fue no probabilística intencionada (Hernández et al., 2014) conformada por 16 participantes previamente conformados de la categoría sub 14 del clubTalentos Envigado (Colombia).

\section{Criterios de inclusión}

Para elegir los deportistas se establecieron criterios de inclusión; jugadores de la categoría sub 14, experiencia federado de al menos un año, firma del asentimiento informado por parte de los menores y firma del consentimiento informado por parte del representante legal. 


\section{Criterios de exclusión}

J ugadores con antecedentes de trastornos de aprendizaje no controlados, antecedentes de trastornos osteomusculares agudos y no asistir al día de las eval uaciones.

\section{Procedimiento}

El presente estudio analizó la adaptación al contexto táctico en términos ofensivos, por medio del principio de situación el cual se refiere a la respuesta táctica idónea que debe ejecutar el jugador analizado y el principio de aplicación que representa la ejecución táctica que realiza el jugador. Esto en relación con los principios ofensivos analizados mantener la posesión de la pelota tres minutos. El juego estuvo conformado por dos equipos de cinco jugadores cada uno, asignados al azar por medio de un software (Epidat 3.1). Este se realizó en un espacio de $40 \times 20 \mathrm{~m}$, un área de meta de $3 \times 4 \mathrm{~m}$ y con porterías de 140x105 cm (Gutiérrez, 2008), cada equipo tuvo portero (Figura 1). El test inició desde el centro del campo; el saque y el campo de juego fue elegido al azar (moneda lanzada al aire por el único árbitro); estuvo prohibido el pase al portero con penalización de saque de banda al equipo contrario; se real izó cobros de tiro de esquina con reinició producto de anotación desde la mitad del campo. Además, se utilizaron ocho balones, se asignaron recogebolas en las zonas laterales del campo y cerca de las porterías. (1A), atacar la portería contraria (2A) y disparar a portería (3A) (Bayer, 1986).

La observación se inició con la identificación de la unidad de toma de decisión (UTD) que sucede cuando un jugador realiza una acción técnico-táctica con la aplica ción de un principio o ejecuta un nuevo elemento técnico-táctico aplicando otro principio o cada cuatro segundos en caso que no haya ocurrido las anteriores. Posteriormente, se observó el principio de situación y el principio de aplicación codificando la toma

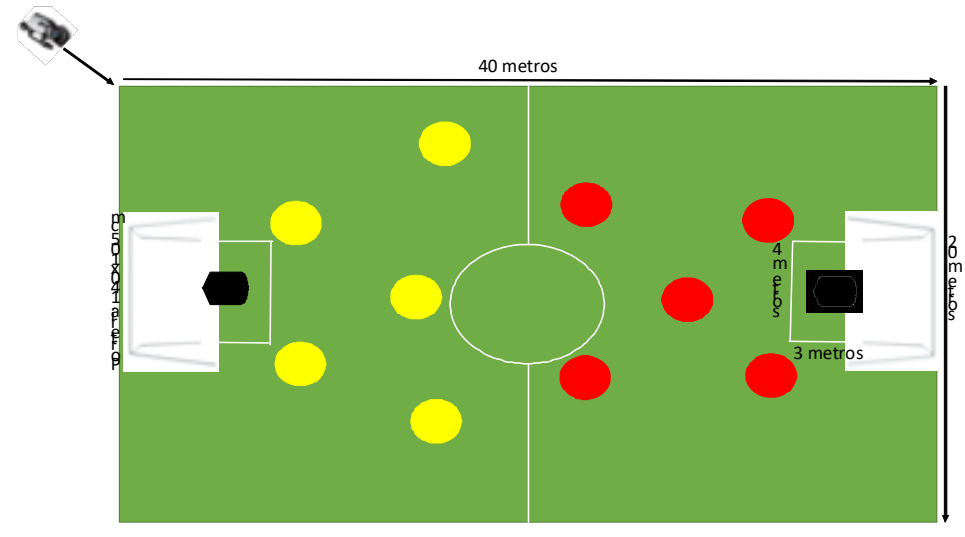
de decisión del deportista con el número 1, cuando fue correcta y 0 cuando fue incorrecta (Gutiérrez, 2008). Los criterios para codificar el principio de situación del jugador atacante que tiene la posesión del balón (JAcB) se pueden observar en laTabla 1.

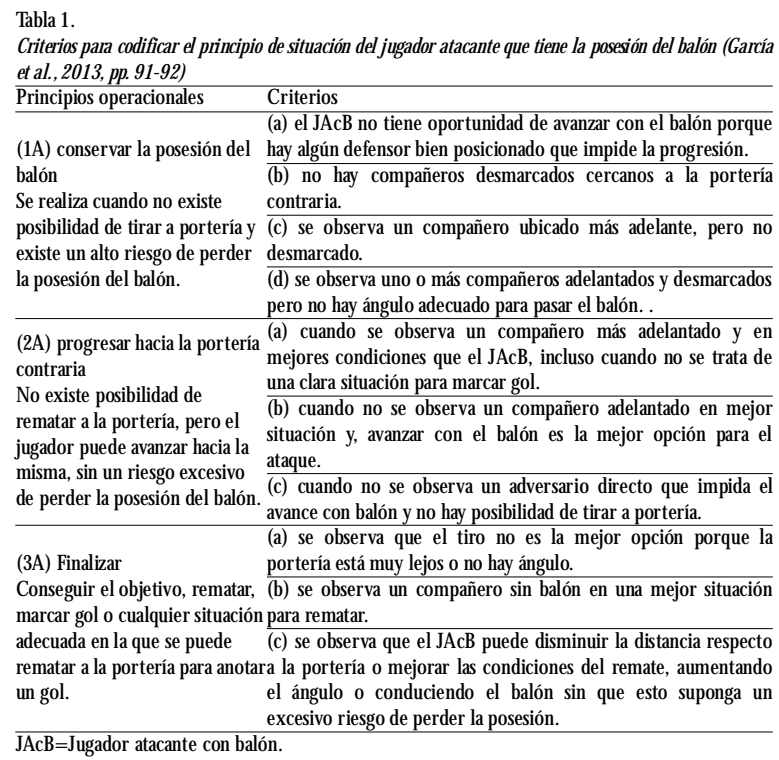

El test consistió en un juego de dos tiempos con duración de cuatro minutos cada uno y un entretiempo de

\section{Instrumentos}

Los datos sociodemográficos como estrato socioeconómico, año escolar cursado, edad, experiencia deportiva y experiencia federada fueron obtenidos por medio de una encuesta prediseñada. En cuanto a las va riables antropométricas, la masa, el porcentaje de grasa, el Índice de Masa Corporal (IMC) fueron tomadas por medio de una báscula de bioimpedancia (HBF-516, OMRO N, Japón) con una precisión de .1 Kg para la masa y $1 \%$ para el porcentaje de grasa. Además, la talla se tomó con una cinta métrica (FIBER-GLASS) de 1.5 m. Estos datos fueron obtenidos teniendo en cuenta los protocolos recomendados por la Sociedad Internacional para el Avance de la Kinantropometría (ISAK) (Stewart et al., 2011). Se utilizó una cámara de vídeo (Hero 5 Black, GoPro, California) para la grabación de los videos que posteriormente fueron descargados a un computador y almacenadas en Google Drive.

Las variables de rendimiento táctico se midieron por medio de la Herramienta de Evaluación del Rendimiento de Juego (HERJ) validada en niños de siete a 14 años de edad (Alpha de Cronbach= .97) (García et al., 2013). Este instrumento analiza y discrimina cada toma 
de decisión desarrollada por el deportista desde el punto de vista táctico, evaluando el comportamiento decisional y ejecución de la acción técnico-táctica.

\section{Control de los sesgos}

Los sesgos de selección, se redujeron mediante el establecimiento de criterios de selección (inclusión y exclusión) para tratar de homogenizar la muestra. Además, se realizó una prueba piloto con el fin de advertir las dificultades que pudieran aparecer, tales como: el tiempo y el espacio para la adecuación de los instrumentos, la ubicación de la cámara, explicación e interiorización del juego por parte de los participantes. Además, se capacitaron los eval uadores para disminuir los sesgos de información y se utilizaron instrumentos válidos y confiables. Final mente, en los sesgos de confusión se midieron variables como la edad, la experiencia deportiva federada y la experiencia deportiva, también se realizó recomendaciones de protocolos de descanso y nutrición a los participantes antes del test.

\section{Análisis estadístico}

Para las variables continuas se realizó la prueba de normalidad Shapiro W ilk $(n<30)$. Los datos con distribución normal ( $p>.05)$, se resumen con media, desvia ción estándar, valor mínimo y máximo. Los datos con distribución no normal $(p<.05)$, se resume con media nas y rangos intercuartílicos. Las variables cualitativas se resumen con porcentajes, frecuencia e intervalo de confianza. Para lo anterior, se utilizó el paquete estadístico Versión 23 IBM Statistical Package for the Social Sciences (Illinois).

Se realizó un análisis bivariado con relación a las acciones de la adaptación al contexto táctico del primer y segundo tiempo mediante la prueba M cNemar para determinar diferencias de proporcionesemparejadascon el software Epidat 3.1 (Xunta de Galicia). Todos los aná lisis se realizarán con un valor alfa $<.05(p<.05)$ y una confiabilidad del $95 \%$.

\section{Análisis de fiabilidad de las observaciones}

Los evaluadores fueron dos estudiantes del último semestre de Entrenamiento Deportivo de la U niversidad de Antioquia con experiencia deportiva superior a 10 años que fueron capacitados con el HERJ; para ello, se realizó una prueba piloto antes de comenzar los aná lisis de los vídeos. Para este análisis de vídeos se impuso que los evaluadores sólo analizarían tres horas por día, descansando 15 minutos por cada hora de anál isis, esto para evitar la fatiga cognitiva.
Para la fiabilidad de las observaciones se presentó una nueva evaluación al eatoria del 20\% de las UTD, una cantidad superior a la recomendada por la literatura (Tabachnick \& Fidell, 2007), estas fueron asignadas al azar por medio del software EPIDAT 3.1. Este procedimiento se realizó con un intervalo de 20 días desde que se elaboró el primer análisis de datos sobre la toma de decisión, cercano al tiempo recomendado (Robinson \& 0 'Donoghue, 2007). Para el análisis de fiabilidad, se aplicó el coeficiente de Kappa (Landis\& Koch, 1977), el cual refleja la concordancia inter-observador y puede ser calculado en tablas de cual quier dimensión, siempre y cuando se contrasten dos observadores (Cerda \& Villarroel, 2008, p. 4) también representa la proporción de acuerdos observados (Abraira, 2001, p. 248). Se verificó la fiabilidad inter-observador con un acuerdo del $93 \%(k=.93)$, mientras que en el análisis intraobservador obtuvo una concordanciadel $100 \%(k=1.0)$; ambos análisis con una fuerza de concordancias casi perfectas (Landis \& Koch, 1977).

\section{Aspectos éticos}

Los participantes y representantes fueron informados de la participación en el estudio, sobre sus riesgos y beneficios. Los jugadores fueron informados al derecho que tenían de participar del estudio; y el hecho de no participar no tendría ningún tipo de represalias en su contra en el clubTalentos de Envigado. El estudio garantizó el respeto a la autonomía, la dignidad y la intimidad delosparticipantes. Según la Resolución 008430 de 1993 del Ministerio de Salud de Colombia artículo 11 numeral b. Los riesgos a los cuales fueron sometidos los individuos están definidos como riesgo mínimo, considera dos como riesgos, los propios de la práctica deportiva. Cada participante y representante firmaron tanto el asentimiento y como el consentimiento informado.

\section{Resultados}

\section{Flujograma de participantes}

En el flujograma se observa una muestra de 16 sujetos, de los cuales tres fueron excluidos al no asistir el día que se tenía prevista la realización del test, por otra parte, dos jugadores no fueron eval uados debido a que su rol desempeñado en el test fue de guardametas y el protocolo no evalúa dicha posición, finalmente un juga dor no fue evaluado por el tiempo y el espacio. En la evaluación antropométrica dos sujetos fueron excluidos (inasistencia y negación las pruebas) (Figura 2). 


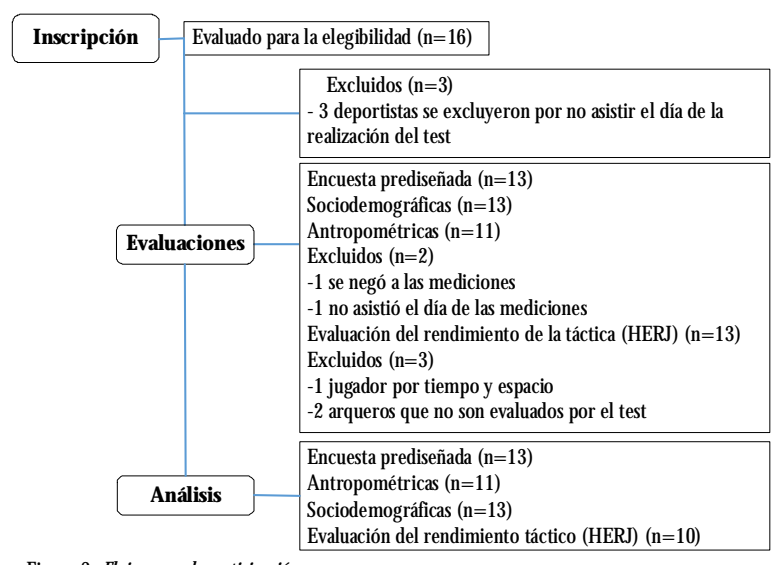

Figura 2. Flujograma de participación

\section{Análisis univariado}

Las variables sociodemográficas cualitativas se resumieron con frecuencias y porcentajes. En laTabla 2 se observa que el estrato socioeconómico con mayor frecuencia fue medio bajo con un total de ocho sujetos $61.5 \%$. Por otra parte, el grado escolar con mayor porcentaje fue noveno con un $61.5 \%$ donde la gran mayoría tienen 14 años.

\begin{tabular}{lcc}
$\begin{array}{l}\text { Tabla } 2 . \\
\text { Variables sociodemográficas }\end{array}$ & & \\
\hline Variable & Frecuencia & Porcentaje \\
Estrato socioeconómico & & 7.7 \\
\hline 1=Bajo-bajo & 1 & 7.7 \\
2=Bajo & 1 & 61.5 \\
3=M edio-bajo & 8 & 23.1 \\
4=M edio & 3 & \\
Grado o nivel de escolaridad & & 7.7 \\
\hline Séptimo & 1 & 30.8 \\
Octavo & 4 & 61.5 \\
Noveno & 8 & \\
\hline
\end{tabular}

Las variables antropométricas de la talla, la masa, el índice de masa corporal y el porcentaje de grasa fueron resumidas con valor mínimo, valor máximo, media y desviación estándar, mientras que la edad, se resume con valor mínimo, máximo, mediana y rango intercuartílico, esto de acuerdo a la distribución de los datos.

En la Tabla 3, se puede observar que la talla tiene una diferencia de $10 \mathrm{~cm}$ entre el valor mínimo y máximo. Para la masa se encontró una media de $55.29 \mathrm{~kg}$ ( $D E=.521$ ), en el caso IMC se halló unamedia de 20.32 $\mathrm{Kg} / \mathrm{m}^{2}(\mathrm{DE}=1.46)$, para el porcentaje de grasa se obtuvo un valor mínimo de 6.1 y máximo de 17.2, en cuanto a la edad, se encontró una mediana de 14 años $(\mathrm{R} I=1.0)$.

Tabla 3.

Variables antropométricas

Variables $\quad$ M Mínima Máxima Media DE Mediana RIC

$\begin{array}{llllll}\text { Tall } a(\mathrm{~cm}) & 11 & 156 & 176 & 165 & .063\end{array}$

$\begin{array}{llllll}\text { Masa }(\mathrm{kg}) & 11 & 44.7 & 62.9 & 55.29 & 5.21\end{array}$

$\begin{array}{llllll}\text { IMC }\left(\mathrm{Kg} / \mathrm{m}^{2}\right) & 11 & 18.8 & 23.2 & 20.32 & 1.46\end{array}$

$\begin{array}{lllllll}\text { Porcentaje de grasa } & 11 & 6.1 & 17.2 & 10.48 & 3.73\end{array}$

$\begin{array}{llcccc}\text { Experiencia deportiva } & 13 & 5 & 10 & 7.84 & 1.34\end{array}$

$\begin{array}{llllllll}\text { Edad (años) } & 13 & 13 & 14 & & & 14 & 1\end{array}$

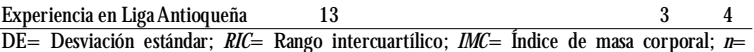
Tamaño de la muestra.
Del total 20 UTD del principio $1 \mathrm{~A}$ en el primer tiempo del test; se obtuvo una efectividad total del $70 \%$ (IC95\%=45.72; 88.10). En el análisis se observó que tres jugadores que lograron un rendimiento del $100 \%$ de efectividad en la toma de decisión. En el análisis de la toma de decisión del principio $2 \mathrm{~A}$ en 45 UTD, se obtuvo una efectividad total del $68.88 \%$ (IC95\% $=54.25$; 83.52), en esta se observa tres participantes con una efectividad del $100 \%$. En el principio $3 \mathrm{~A}$ se obtuvo un análisis de ocho unidades de toma de decisión y se presenta una efectividad total del 75\% (IC95\% = 34.91; 96.81) donde cuatro jóvenes presentaron una efectividad del $100 \%$. En los datos registrados del primer tiempo se obtuvo una efectividad total representada por el $69.86 \%$ (IC95\% = 58.65; 81.07) en las 73 UTD. En relación con las 16 situaciones excepcionales reportadas en el primer tiempo, se obtuvo que el $12.50 \%(n=2)$ fueron situaciones de balón dividido y el $87.50 \%$ ( $n=$ 14) no hubo aplicación de principio.

En el segundo tiempo se registró 19 unidades de tomade decisión con una efectividad de $68.42 \%$ (IC95\% $=43.45 ; 87.42)$ para el principio $1 \mathrm{~A}$ donde cinco sujetos logran el máximo con un $100 \%$ de efectividad. En el principio 2A se obtuvo un total de 37 UTD representando el 94.59\% (IC95\% = 81.80; 99.33) de efectividad, manifestando el 100\% de efectividad por parte de siete sujetos. Un total de cinco unidades de toma de decisión representadas en los cuatro sujetos con efectividad del $100 \%$ (IC95\% $=47.81 ; 100)$ en el principio de finalización. Se observó que el $20 \%$ de los sujetos presentó seis UTD y de las 61 unidades de toma de decisión que se presentaron en el segundo tiempo, se obtuvo un $86.89 \%$ (IC95\% $=77.59 ; 96.17$ ) de efectividad en los principios operacionales. Un total de 41 situaciones excepcionales, de las cual es el $19.51 \%(n=8)$ son acciones de balón dividido y el $80.49 \%(n=33)$ la no aplicación de principio en el segundo tiempo.

Se observaron un total 191 unidades de análisis, de las cuales, 57 representan las situaciones excepcionales de balón dividido y no aplicación de principio. Se obtuvo un $82.46 \%(n=47)$ de situaciones excepciones en las queno se aplicó ningún principio, mientrasqueel $17.54 \%$ $(n=10)$ fueron balones divididos (Tabla 4).

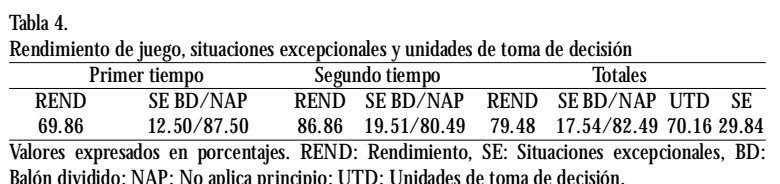

Se observa el rendimiento de cada tiempo y total en la Figura 3, donde se registró en el primer tiempo 
un total de 89 acciones, mientras que en el segundo hubo un ascenso en el número de UTD (102), y finalmente se obtuvo un promedio del porcentaje de la efectividad de todos los principios (principio 1A, 2A y $3 \mathrm{~A}$ ). Se resalta el segundo tiempo porque presenta un mayor rendimiento $(86.89 \%)$ comparado con el primer tiempo (69.86\%); además se obtuvo el rendimiento total de ambos tiempos (79.48\%).

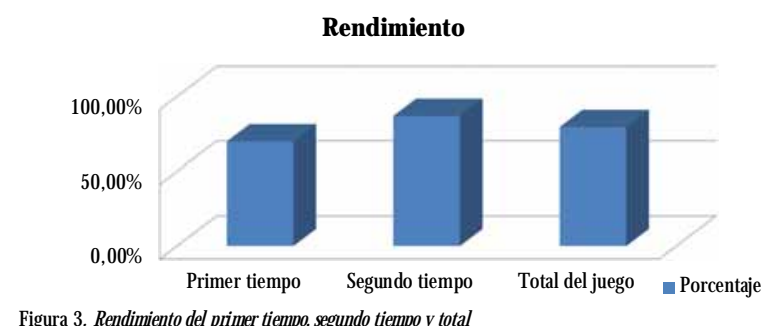

\section{Análisis bivariado}

Se realizó un análisis bivariado de la efectividad de los principios de conser var el balón (1A), progresar (2A) y finalizar ( $3 A$ ) entre el primer tiempo comparado con el segundo tiempo mediante el estadístico M CNemar para determinar diferencias de proporciones emparejadas. En la Tabla 5, se puede observar que el rendimiento en el principio de conservar tuvo un descenso en el segundo tiempo con relación al primer tiempo ($2.26 \%)$ sin que esto implique diferencias estadísticamente significativas (IC95\% $=-6.38 ; 36.46$; $p=.16)$.

Por otra parte, se encontró que en el principio de progresar hubo un ascenso considerable en el segundo tiempo en comparación con la primera parte (37.33\%), con diferencias estadísticamente significativas (IC95\% = 41.39; 9.82; $p<.01$ ).

En cuanto a la efectividad del contexto táctico para finalizar, no se contó con valor $p$ ni intervalo de confianza del $95 \%$, debido a que no fue posible calcularlos en la tabla cruzada 2vs2 de M cN emar porque uno de los da tos fue cero y el programa estadístico no calcula datos con esta condición, se resalta que existe una diferencia del $33.33 \%$ entre la efectividad del contexto táctico para finalizar del segundo tiempo con relación al primer tiempo y genera una diferencia importante desde

\footnotetext{
Tabla 5.

Análisis bivariados: Diferencias entre el primer tiempo y segundo tiempo en la adaptación al contexto táctico Adaptación al contexto \%Primer \%Segundo \% diferencias entre Intervalo de McNemar Valor táctico tiempo tiempo segundo tiempo- Confianza del Ji-cuadrado $p$ \begin{tabular}{lllccc}
\hline Conservar el balón (1A) & 70 & 68.42 & -2.26 & $-6.38 ; 36.46$ & .16
\end{tabular} $\begin{array}{lllllll}\text { Progresar (2A) } & 68.88 & 94.59 & 37.33 & 41.39 ; 9.82 & 8.16 & .01 *\end{array}$ $\begin{array}{lcccccc}\text { Finalizar (3A) } & 75 & 100 & 33.33 & & & \\ \text { Efectividad total } & 69.86 & 86.89 & 24.37 & 11.08 ; 35.18 & 72.39 & .01 *\end{array}$ \begin{tabular}{lrrrrrr} 
Efectividad total & 69.86 & 86.89 & 24.37 & $11.08 ; 35.18$ & 72.39 & $.01 *$ \\
\hline * Diferencias estadísticamente muy significativas entre la efectividad del contexto táctico del
\end{tabular} segundo tiempo y primer tiempo $p<.05$.
}

el punto de vista práctico; finalmente, en la efectividad total se encontró una diferencia entre el segundo tiempo y el primer tiempo del $24.37 \%$ con diferencias estadísticamente significativas (IC95\% = 11.08; 35.18; $p<.01)$.

\section{Discusión}

El objetivo del estudio fue describir el nivel de adaptación al contexto táctico basado en los principios operacionales propuestos por Bayer (1986): conservar (1A), progresar el balón (2A) y finalizar (3A), en juga dores de 14 años en un partido de fútbol modificado (5+Portero vs $5+$ Portero) con duración de ocho minutos dividido en dos tiempos, utilizando el instrumento HERJ y establecer diferencias de rendimiento entre el primer y segundo tiempo del juego.

En el análisis descriptivo se observó que los jugadores realizaron un total de 191 acciones, divididas en 83 acciones en el primer tiempo y 102 acciones en el segundo tiempo; también, se presentó 57 situaciones excepcionales totales, presentadas en su mayoría cuando no hubo aplicación de principios (47), esto se debe aque el balón salió de la zona de juego y en algunas ocasiones hubo tardanza en disponer el balón nuevamente en el juego. De esta manera, los deportistas realizaron 39 UTD parala aplicación del principio $1 A$, donde se obtuvo una efectividad del $69.21 \%$ (IC $95 \%=53.46 ; 84.99)$. También, se realizaron un total 82 UTD en el principio $2 \mathrm{~A}$, representando una efectividad del $81.73 \%$ (IC95\% = 71.30; 89.67), mientras que en el principio 3 A sólo se registraron 13 UTD con una efectividad del $87.5 \%$ (IC95\%= 54.55; 98.07). El análisis del contexto táctico en la efectividad total de los principios operacionales de conservar el balón, progresar y finalizar (Bayer, 1986) en ambos tiempos los deportistas presentaron un desempeño superior al $71 \%$.

La comprensión de los principios por parte de los jugadores según Costa et al., (2009) ayuda a la estructuración y desarrollo de las acciones técnico-tácticas del juego pretendidas. Posiblemente el nivel de rendimiento en la adaptación al contexto táctico en los jugadores de esta categoría y de acuerdo a Garganta \& Pinto (1997), loscuales clasifican dicho rendimiento por modelos de juego que va desde modelo tipo uno hasta modelo tipo cinco, se puede considerar que este grupo probablemente se encuentre en el modelo tipo cuatro, el cual tienen características de juego dinámico, orientado y jugadores centrados en conseguir la finalidad «el gol». 
En el análisis realizado para comparar el nivel de rendimiento entre el primer y segundo tiempo, se puede observar que los deportistas mejoraron la efectividad en la adaptación al contexto táctico en el segundo tiempo, esto en los principios de progresar y finalizar, además del rendimiento total (RT), donde se obtuvo una evolución del (RT 37.33\% IC95\%= 41.39; 9.82, $2 \mathrm{~A} 33.33 \%$, IC $95 \%=$ sin dato y $3 \mathrm{~A} 24.37 \%$ IC $95 \%=$ $11.08 ; 35.18)$ mientras que el principio de conservación tuvo un descenso de 2.26\% (IC95\% = -6.38; 36.46). Respecto a estos resultados, esto es coherente y rela cionado con lo que menciona Blomqvist et al., (2005) que estudiaron la toma de decisión de jóvenes deportistas entre los 12 y 15 años, concluyendo que a esta edad los jugadores ya tienen una comprensión y capacidad de juego en los principios ofensivos y defensivos, en este caso los principios operacionales del juego.

Además, de acuerdo con los estados de desarrollo cognitivo de Piaget \& Cook, (1952), se advierte que este grupo se encuentra en el periodo de operaciones formales, el cual va desde los 11 años en adelante; en dicho periodo, se tiene un pensamiento racional e inductivo para afrontar cualquier situación que se presente. Según Castilla (2014) en esta etapa los adolescentes cuenta con un razonamiento hipotético-deductivo, eso ayuda a los deportistas a realizar predicciones sobre hechos hipotéticos o futuros, permitiendo a los mismos tener una mayor capacidad al momento de resolver problemas que constantemente se presentan en el terreno de juego y que exigen una gran demanda cognitiva y motriz. Además, la toma decisión está rela cionada con la capacidad de utilización de la memoria de trabajo y la memoria a largo plazo (López, 2008).

De acuerdo a lo anterior, se logra observar que los deportistas mejoran el rendimiento en los principios $2 \mathrm{~A}, 3 \mathrm{~A}$ y $\mathrm{RT}$ de la adaptación al contexto táctico en el segundo tiempo, posiblemente esto sucedió debido a la experiencia previa que se generó en el primer tiempo por parte de los individuos que involucró el conocimiento del espacio, sus compañeros y sus rivales. Por ende, aprovechando su capacidad y dominio del juego en el segundo tiempo pudieron tener un mayor desempeño adaptándose a estos contextos al momento de la toma de decisión. Por otro lado, se resalta que los deportistas tuvieron un descanso de tres minutos en el que algunos jugadores tomaron el liderazgo y discutieron desde el punto de vista estratégico como podrían mejorar el rendimiento en el segundo tiempo, cabe señalar que los deportistas no tuvieron ningún tipo defeedback por parte del entrenador 0 evaluadores.
Los resultados del estudio contrastan con los hallazgos de González-Víllora et al. , (2012), los cuales evaIuaron deportistas de ocho años del Albacete Balompié por medio de juego modificado de 2vs2 mediante la misma herramienta (HERJ). Los resultados, fueron mayores en el principio de conservar $86.88 \%$ en comparación con el presente estudio $(69.21 \%)$, mientras que losprincipios de progresar $81.73 \%$ y finalizar $87.5 \%$ son valores superiores en contraste con los encontrados en el estudio ( $73.49 \%$ y $72.42 \%$, respectivamente). Estos resultados podrían darse debido a la experiencia y la diferencia de edad que se tiene entre los deportistas (14 años vs ocho años) y la estructura de juego en la evaluación (2vs2; 5vs5), además el estudio no utilizó porteros.

De la misma manera, O livares et al., (2011) evaIuaron deportistas de ocho años del Albacete Balompié en un juego 3vs3 de representación donde analizaron los principios de conservar, progresar y finalizar y otro de exageración donde se mantuvo el análisis sólo del principio de conservación mediante el HERJ. Para el análisis, se tuvo en cuenta solo los valores hallados en el juego de representación, debido a que estetenía en cuenta los tres principios operacionales. Se resal ta que los deportistas fueron evaluados en un campo con dimensiones de $32 \times 22$ además, se limitó el número de toques (cuatro) a cada jugador y no utilizaron portero, los resultados demuestran una efectividad en el principio $1 \mathrm{~A}$ del $84.01 \%$; en el principio $2 \mathrm{~A}$ del $82.91 \%$ y en el $3 \mathrm{~A}$ de $81.20 \%$; siendo estos valores superiores al presente estudio en los principios de conservar y progresar, no obstante se obtuvo valores superiores en el principio de finalizar (87.5\%). Esto posiblemente por la diferencia en la evaluación, las dimensiones del terreno (dos metros mayor el ancho del campo); a pesar, que la cantidad de participantes fue menor en el juego. 0 tro factor, que pudo influir fue la condición o restricción en el juego. Además, los jugadores del antecedente fueron escogidos por entrenadores de acuerdo a su nivel de juego, donde se escogieron sólo jugadores con un nivel de pericia medio-alto que proclaman una puntuación superior o igual a siete en una escala de cero a diez, mientras que este estudio no realizó una muestra estratificada por nivel de juego y finalmente no utilizaron porteros.

Los resultados obtenidos en esta investigación comparados con el estudio Gutiérrez \& García-ópez, (2012) con futbolistas de 11 y 12 años, demuestran mejores resultadosen cadaprincipio ( $1 A=62.97 \%, 2 A=70.08 \%$, $3 A=55.41 \%)$. El estudio en mención obtuvo un rendimiento total del $66.8 \%$ mientras que en el presente 
estudio, reporto valores mayores en el rendimiento total $(79.48 \%)$ con una diferencia porcentual de $18.98 \%$, demostrando así una mayor efectividad en la adaptación al contexto táctico, tal vez por la diferencia de la edad.

Del Campo et al., (2011) estudiaron la diferencia que tenían en la toma de decisiones entre deportistas expertos y novatos (educación física) entre los ocho a 15 años, los jugadores expertos fueron integrantes del Albacete Balompié, y los novatos fueron seleccionados estudiantes de educación física sin ninguna experiencia en juegos de invasión y ninguna experiencia en competencias en el deporte; estos fueron evaluados por medio del HERJ. En laTabla 6, se observa que los deportistas novatos mostraron un rendimiento global por categoría así sub 9 del $67.4 \%$, sub 11 del $76 \%$, sub 13 del $66.8 \%$ y sub 15 del $7703 \%$, siendo superiores los deportistas del descritos en todas las categorías de nova tos (79.48\%). Mientras que los datos con los jugadores expertos presentaron un rendimiento global así: sub 9 del $78.2 \%$, sub 11 del $85.5 \%$, sub 13 del $88.5 \%$ y sub 15 del $94.3 \%$. Se puede notar que los resultados de estos deportistas son superiores en todas las categorías a excepción de la categoría sub 9. Esto puede deberse a que los jugadores expertos pertenecen a las reservas de proyección del equipo profesional (Albacete Balompié) donde probablemente se hace proceso riguroso de captación y selección de jugadores, mientrasqueen el equipo objeto de estudio puede darse dicho proceso en un nivel menor, porque el club de fútbol (Tal entos Envigado) no cuenta con categoría pre-benjamín (Sub 7-8) y la capta ción de jugadores se realiza anualmente al inicio del año, donde los deportistas son elegidos de acuerdo al análisis subjetivo de las habilidades y capacidad de juego que perciben los entrenadores. Cabe resaltar que el Albacete Balompié garantiza todo el proceso de forma ción del deportista desde el fútbol base hasta el alto rendimiento en la misma institución, en tanto el club objeto de estudio es amateur y no garantiza todo el proceso en el mismo. Además, se resalta que los deportistas del Albacete basan sus entrenamientos en un enfoque centrado en la táctica, ayudando a estos a tener una mejor adaptación a los contextos tácticos, generando una mayor experiencia en los juegos de invasión (del

\begin{tabular}{|c|c|c|c|c|}
\hline \multicolumn{5}{|c|}{ Comparación de la adaptación al contexto táctico: Presente estudio vs Estudio de Del campo et al., (2011) } \\
\hline Sub 14 Presente estudio & 69.21 & 81.73 & 87.5 & 79.48 \\
\hline Sub 9 expertos* & 86.9 & 73.5 & 72.4 & 78.2 \\
\hline Sub 9 novatos** & 65.7 & 68.1 & 78.5 & 67.4 \\
\hline Sub 11 expertos & 90.4 & 85.7 & 73.6 & 85.5 \\
\hline Sub 11 novatos & 80.6 & 74.6 & 65.2 & 76 \\
\hline Sub 13 expertos & 77.8 & 90.7 & 75 & 88.5 \\
\hline Sub 13 novatos & 63 & 70.1 & 55.4 & 66.8 \\
\hline Sub 15 expertos & 85.5 & 99.3 & 94.4 & 94.3 \\
\hline Sub 15 novatos & 68.4 & 79.2 & 82.8 & 77.3 \\
\hline
\end{tabular}

Campo et al., 2011).

Finalmente, en las últimas décadas la enseñanza de los deportes colectivos ha sufrido grandes cambios en las metodologías utilizadas, desde las más tradicionales orientadas a la adquisición de los gestos técnicos, hasta metodologías más activas basadas en la adquisición de habilidades tácticas que brindan la posibilidad de construir propios aprendizajes(Abad et al., 2013; A rias et al., 2016), esto basado en los sistemas dinámicos, la psicologíaecológicay lasteorías constructivistas(Serra-O livares $\&$ Garcia-Rubio, 2017). Dicho esto y con base a los resultados del estudio se recomienda a los entrenadores la utilización de enfoques centrados en el juego y en el jugador como Teaching $G$ ame for Undersatnding (Bunker \& Thorpe, 1982), Tactical Games Approach (Mitchell et al., 2006), Modelo Didáctico de Competencias deAcción de Juego (Valencia \& Arias, 2015) que estimulan en mayor medida las habilidades tácticas con la idea de formar jugadores con capacidad de resolver problemas (López et al., 2010; López, 2008). 0 tra posibilidad es la pedagogía no lineal como método de enseñanza de comportamiento tácticos para la adaptación del jugador a situaciones reales de juego donde se manipula los cuatro principios pedagógicos: complejidad táctica, representatividad de la tarea, exageración y muestreo (Barrero \& Lazarraga, 2020; Cantos \& Hernández, 2019). Siendo necesario realizar estudios experimentales que confirmen los efectos de entrena miento de las metodologías mencionadas principalmente en el aspecto táctico.

\section{Conclusiones}

En el principio 1A se obtuvo una disminución del rendimiento en el segundo tiempo comparado con el primer tiempo sin diferencias significativas.

En el principio 2A los sujetos tuvieron un mejor rendimiento en el segundo tiempo comparado con el primer tiempo con diferencia estadísticamente significativa.

En cuanto al principio 3A se presentó una efectividad completa en el segundo tiempo encontrando una diferencia práctica al ta comparado con el primer tiempo.

En términos generales, hubo mejor adaptación al contexto táctico en el segundo tiempo comparado con el primer tiempo con una diferencia estadísticamente muy significativa en los jugadores.

Los jugadores del estudio deben tener una participación alta en la conservación del balón en el primer tiempo e intentar sacar ventaja en el segundo tiempo, 
donde tienen mayor rendimiento en la adaptación al contexto táctico en los principios de progresión y finalización.

En el proceso de entrenamiento se debe promover el desarrollo de las habilidades tácticas focalizando la atención en los principios tácticos operaciones desde el primer segundo de juego.

\section{Recomendaciones}

Se sugiere replicar el estudio, aumentando el tama ño de la muestra e incluyendo diferentes categorías para establecer niveles en la adaptación táctica de acuerdo a la edad y ajustar los programas de entrenamiento en coherencia con su nivel de juego.

\section{Limitaciones}

El tamaño de las porterías y el área, tuvieron que ser adaptados de acuerdo a los lineamientos del HERJ. Los factores ambientales, como la calidad del aire en la ciudad de Medellín y posteriormente la pandemia producto del virus SARS-CoV-2 disminuyó la cantidad de participantes prevista en el estudio, siendo la principal limitación del estudio.

\section{Agradecimientos}

Los autores agradecen la colaboración del club Ta lentosEnvigado (Colombia) y sus deportistas por la participación en el estudio.

\section{Referencias}

Abad Robles, M.T. (2010). La formación del entrenador en el proceso de enseñanza-aprendizajedejóvenesfutbolistas [TesisD octoral, Universidad deH uelva]. Recuperado dehttps:/ / www. researchgate. net/ profile/Abad_Robles/publication/ 279417288 _La_formacion_del_entrenador. e n_el_proceso_d e_ensenanza. aprendizaje_de_jovenes_futbolistas/links/ 5598e2bb08ae793d137e22ce. pdf

Abad Robles, M. T., Giménez Fuentes-Guerra, F. J., \& Robles Rodríguez,J. (2013). Fundamentospedagógicos delaenseñanza comprensivadel deporte: Unarevisión de laliteratura. Cultura, Ciencia y Deporte, 23, 137-146. https:/ / doi.org/ 10.12800/ ccd. v8i23.300

Abraira,V. (2001). El índicekappa. Semergen-M edicina deFamilia, 27(5), 247-249.

Aguero, M., Nelson, R., Gómez, B. \& \& Traba, D. (2015). Replantea miento de los elementoscomponentes de latáctica en el fútbol. Efdeportes. com, 1(1).

Amado O rtiz, S., \& Vivas Rodríguez, J. F. (2014). El desarrollo de conceptostácticos, con jóvenes de 14 y 15 añosdeedad en la academia de fútbol Arnoldo I guarán de Bogotá. [Tesis de pregrado, U niversidad Libre]. Recuperado de https:/ / repository.unilibre. edu.co/ bitstream/hand le / $10901 / 8537 /$ Proyecto $\% 20$ tactica $\% 20$ en $\% 20$ el $\% 20$ f $\%$ c $3 \%$ batbol. pdf?sequence=1\&isAllowed=y

Américo, H. B., Kowalski, M., Cardoso, F., Kunrath, C. A., GonzálezVíllora, S. \&Teoldo, I. (2017). Differencein declarativetactical knowledge between $\mathrm{U}-11$ and $\mathrm{U}-15$ soccer players. H uman Movement Special Issues, 2017(5), 25-30.

Arias, A. G. , Arroyo, M. P. M. , Rabaz, F. C., Domínguez, A. M. \& \& Álvarez, F. D.V. (2016). Manipulación deloscondicionantesdela tareaen Educación Física: U napropuesta desde la pedagogíano lineal. Retos. N uevasTendencias en Educación Física, D eportey Recreación, 29, 22-27.

Arrieta, P., Castellano, J., Guridi, I., \& Echeazarra, I. (2017). Efectos de un programa basado en juegos reducidos sobrela condición físicadejóvenesjugadores de fútbol. RICYDE. Revista Internacional de Ciencias del Deporte. doi: 10.5232/ ricyde, 13(50), 370-380. https:/ / doi. org/ 10.5232/ ricyde2017.05004

Barrero, A. M ., \& Lazarraga, P. C. (2020). El diseño de tareas de entrenamiento en el fútbol desdeel enfoque de la pedagogíano lineal. Retos: $N$ uevasTendencias en Educación Física, D eportey Recreación, 38, 768-772.

Bayer, C. (1986). La Enseñanza del osjuegos deportivoscol ectivos: Baloncesto, futbol, balonmano, hockey sobre hierba y sobre hielo, rugby, balonbolea, waterpolo. Hispano Europea.

Bettega, O. B., Scaglia, A. J. , do N ascimento, J. V., Godoy, S. J. I. , \& Galatti, L. R. (2018). 0 ensino da tática eda técnica no futebol: Concepção de treinadores das categorias de base. Retos: N uevas Tendenciasen Educación Física, D eportey Recreación, 33, 112-117.

Blomqvist, M .,Vänttinen, T., \& Luhtanen, P. (2005). Assessment of secondary school students' decision-making and game-play ability in soccer. Physical Education \& Sport Pedagogy, 10(2), 107-119.

Bunker, D. , \&Thorpe, R. (1982). A M odel for the teaching of games in secondary schools. Bulletin of Physical Education, 18(1), 5-8.

Cantos, J. , \& Hernández, F. J. M. (2019). Pedagogía no lineal como método de enseñanza de los comportamientos tácticos en los deportes de equipo, aplicación al rugby. Retos: N uevasTendenciasen Educación Física, Deportey Recreación, 35, 402-406.

Castilla Pérez, M. F. (2014). La teoría del desarrollo cognitivo de Piaget aplicada en la clase de primaria. [Tesis de grado, Universidad deValladolid]. Recuperado dehttp:/ / uvadoc. uva. es/ bitstream/ $\mathrm{h}$ a nd l e / $10324 / 5844 / \mathrm{T}$ G B.531. pdf?sequence $=1$ \&isAllowed $=y$

Castro, C. A., Árias, A. M ., \& Gracia Díaz, Á. J. (2019). Análisis táctico deportivo con herramientastecnológicas, aplicadasajugadores de fútbol profesional del club Independiente SantaFe. Revista digital:Actividad Física y Deporte, 5(1), 34-50.

Cerda, J. , \&Villarroel Del, L. (2008). Evaluación dela concordancia inter-observador en investigación pediátrica: Coeficiente de Kappa. Revista chilena depediatría, $79(1), 54-58$.

CorreiadaSilva, D., Teoldo daCosta, V., Casanova, F., M anuel Clemente, F., \& Teoldo, I. (2019). Comparison between teams of different ranksin small-sided and conditioned gamestournaments. International Journal of PerformanceAnalysisin Sport, 19 (4), 608-623.

Costa, I. T. D., Garganta, J., Greco, P. J., \& M esquita, I. (2009). Princípiostáticos do jogo de futebol: Conceitose aplicação. M otriz, Rio Claro, 15(3), 657-669. 
DaSilva, R. N. B., Thiengo, C. R., Talamoni, G. A., Lima, M. R., \& Da Costa, I.T. (2015). Desempenho tático de jogadores sub-15 do são paulo futebol clubeapartir do testefut-sat. Educación Física y Deporte, 34(1), 181-199.

del Campo, D. G. D. ,Villora, S. G., Lopez, L. M. G., \& Mitchell, S. (2011). Differences in decision-making development between expert and novice invasion game players. Perceptual and Motor Skills, 112(3), 871-888. http:/ / dx.doi.org/ 10.2466/ 05.10.11.25.PMS. 112.3.871-888

Delgado, J. C. G., Longoria, R. J. N. , Enríquez, O. N. , Luján, R. C., \& Gastélum, G. (2019). Sistemastácticosy resultados de competición del M undial de Fútbol Asociación de Rusia 2018. Retos: N uevasTendencias en Educación Física, D eportey Recreación, 36, 503509.

GarcíaLópez, L. M ., GonzálezVillora, S., Gutiérrez, D., \& Serra, J. (2013). D esarrollo y validación de laherramientade evaluación del rendimiento de juego (HERJ) en fútbol. Sportk, 2 (1), 89-99.

García, S. , Rodríguez, A. \& \& Garzón, A. (2011). Conceptualización deinteligenciatácticaen fútbol: Consideracionesparael desarroIlo deun instrumento de eval uación en campo desdelasfuncionesejecutivas. Cuadernos de Psicología del Deporte, 11 (1), 69-78.

Garganta, J., \& Pinto, J. (1997). La enseñanza del fútbol. En La enseñanza delosjuegosdeportivos (Graca, Amandio-O liveiraJ osé, p. 244). Paidotribo.

Gómez López, M. T., \& Álvaro Alcalde, J. (2002). El tiempo de posesión como variable no determinante del resultado en los partidosdefútbol. Kronos, 1(1), 1-9.

GonzálezVíllora, S., García López, L. M, Pastor Vicedo, J. C., \& Contreras) ordán, O. R. (2011). Conocimiento táctico y tomade decisiones en jóvenes jugadores de fútbol (10 años). Revista de Psicología del Deporte, 20(1), 79-97.

González-Víllora, S., GarcíaLópez, L. M., Gutiérrez-Díaz del Campo, D. , \& Pastor-Vicedo, J. C. (2012). Estudio del rendimiento de juego (2 vs. 2) en jugadores de fútbol con 8 años. Revista de Investigación en Educación, 10(1), 115-126.

Guimaraes, T. (2000). El sistema, la táctica y losjugadores. Magisterio.

Gutiérrez, D., \& GarcíaLópez, L. M. (2012).Assessment of primary school students' decision-making related to tactical contexts. $N$ ew Approachesin Educational Research, 1(1), 7-12.

Gutiérrez Díaz del Campo, D. (2008). Desarrol lo del pensamiento táctico en edad escolar [Doctoral dissertation]. U niversidad de Castillala Mancha

Hernández, R., Fernández, C. \& \& Baptista, P. (2014). M etodología de la investigación (6. ${ }^{\mathrm{a}} \mathrm{ed}$.). Mc Graw-Hill.

Landis, J. R., \& Koch, G. G. (1977). The measurement of observer agreement for categorical data. Biometrics, 159-174.

López, F.A.,Vélez, D. C., León, M.T. M., O rtín, N. U., \& López, M. I. P. (2010). Lamejoradela capacidad de atención selectiva del jugador debaloncesto através delaenseñanza orientadaal aprendizaje táctico. (Enhancing the selective perception of basketball playersthrough tactical learning). Cultura, Ciencia y D eporte, 5(14), 101-108.

López, L. M. G. (2008). Investigación y enseñanzatécnico-tácticaen el fútbol. (Research and Teaching of Techniques and Tactics in Invasion Games. Implementation in Soccer). Cultura, Ciencia y Deporte, 3(9), 161-168.

López, L. M. G., \& del Campo, D. G. D. (2018). Contributions of the GPET to the GPAI: tactical context adaptation and game behaviour. Retos: N uevasTendenciasen Educación Física, D eportey Re creación, 34, 323-328.

Congreso de la República sobre las consideraciones éticas para la investigación con seres humanos., Pub. L. No. Resolución N 008430 (1993).

Mitchell, S., 0 slin, J., \& Griffin, L. L. (2006). Teaching sport conceptsand skills:A tactical gamesapproach (2. ${ }^{\mathrm{a}} \mathrm{ed}$.). H uman Kinetics.

MoralesCastillo,Y., \& M urillo H oyos, E. A. (2013). Origen y evolución delossistemastácticosen el fútbol [Tesis de pregrado, U niversidad del Valle]. Recuperado dehttps:/ / bibliotecadigital univalle edu.co/ bitstream/hand le/ $10893 / 7208 / 3410$ 0430864. pdf?sequence=1\&isAllowed=y

O livares, J. S. , Ayuso, A. P., Vicedo, J. C. P., \&Víllora, S. G. (2020). Propuestade evaluación multidisciplinar del talento dejóvenes futbolistas. Retos: N uevasTendencias en Educación Física, D eporte y Recreación, 38, 40.

Olivares, J. S. ,Víllora, S. G., \& López, L. M. G. (2011). Comparación del rendimiento de juego dejugadores de fútbol de 8-9 añosen dosjuegos modificados 3 contra3. Cuadernosde Psicología del De porte, 11(2), 77-92.

Piaget, . ., \& Cook, M. (1952). Theorigins of intelligence in children (Vol. 8, N úmero 5). International Universities Press NewYork.

Práxedes, A., Del Villar Álvarez, F., Moreno, A., Gil-Arias, A., \& Davids, K. (2019). Effects of a nonlinear pedagogy intervention programme on the emergent tactical behaviours of youth footballers. Physical Education and Sport Pedagogy, 24 (4), 332-343. https:/ / doi. org/ 10.1080/ 17408989.2019.1580689

Riera, J. (2007). Acercadel deportey el deportista. Revista de Psicología del $D$ eporte, $6(1)$.

Robinson, G. , \& O'D onoghue, P. (2007). A weighted kappastatistic for reliability testing in performance analysis of sport. International Journal of PerformanceAnalysis in Sport, Cardiff, 7(1), 12-19.

Serra-O livares, J. , \& Garcia-Rubio, J. (2017). Laproblemáticatácti$\mathrm{Ca}$, claveen el diseño representativo detareas desdeel enfoque de la pedagogíano lineal aplicadaal deporte. RETOS. N uevasTendencias en Educación Física, D eportey Recreación, 32, 270-278.

Sgrò, F. , Bracco, S. , Pignato, S. , \& Lipoma, M . (2018). Small-sided games and technical skills in soccer training: Systematic review and implications for sport and physical education practitioners. Journal of SportsScience, 6(1), 9-19. https:/ / doi.org/ 10.17265/ 2332-7839/2018.01.002

Stewart, A. , M arfell-Jones, M., O Ids, T., \& Ridder, D. H. (2011). ISAK: International standards for anthropometric assessment. International Society forA dvancement of K inanthropometry, Lower Hutt.

Tabachnick, B. G., \& Fidell, L. S. (2007). U sing multivariate statistics (Q uinta). Harper \& Row.

Valencia,W. , \&A rias, E. (2015). El M odelo Didáctico de las competencias de acción de juego, efectos de aprendizaje y validación empírica. Expomotricidad: Instituto U niversitario deEducación Física; Sistema de Revistas dela U niversidad deAntioquia, 1(1), 277-284.

Vergara, J. D. O. (2012). Incidencia de un plan de entrenamiento estructurado a través de juegosmodificadossobre laocupación deespacios libresen fase ofensiva. VIREF Revista deEducación Física, 1(1), 79-94. 